\title{
The RUB cage: respiration-ultrasonic vocalizations-behavior acquisition setup for assessing emotional memory in rats
}

\section{Chloé Hegoburu ${ }^{1,2 *}$, Kiseko Shionoya ${ }^{1,2+}$, Samuel Garcia ${ }^{1,2}$, Belkacem Messaoudi,2, Marc Thévenet ${ }^{1,2}$ and Anne-Marie Mouly,2}

\author{
1 Team "Olfaction: From Coding to Memory," Lyon Neuroscience Research Center, INSERM U1028, CNRS UMR5292, Lyon, France \\ 2 University Lyon 1, Lyon, France
}

Edited by:

Carmen Sandi, Ecole Polytechnique Fédérale de Lausanne, Switzerland

\section{Reviewed by:}

Martine Ammassari-Teule, Consiglio Nazionale delle Ricerche, Italy

Markus Wöhr, Philipps-University of

Marburg, Germany

\section{*Correspondence:}

Chloé Hegoburu, Team "Olfaction:

From Coding to Memory," Centre de

Recherche en Neurosciences de Lyon INSERM U1028, CNRS UMR5292, 50

Avenue Tony Garnier, F-69366 Lyon

Cedex 07, France.

e-mail: chloe.hegoburu@olfac.

univ-lyon 1.fr

\section{${ }^{t}$ Present address:}

Kiseko Shionoya, Max Planck Institute of Biophysics, Max-von-Laue-Strasse 3, D-60438 Frankfurt, Germany
In animals, emotional memory is classically assessed through pavlovian fear conditioning in which a neutral novel stimulus (conditioned stimulus) is paired with an aversive unconditioned stimulus. After conditioning, the conditioned stimulus elicits a fear response characterized by a wide range of behavioral and physiological responses. Despite the existence of this large repertoire of responses, freezing behavior is often the sole parameter used for quantifying fear response, thus limiting emotional memory appraisal to this unique index. Interestingly, respiratory changes and ultrasonic vocalizations (USV) can occur during fear response, yet very few studies investigated the link between these different parameters and freezing. The aim of the present study was to design an experimental setup allowing the simultaneous recording of respiration, USV, and behavior (RUB cage), and the offline synchronization of the collected data for fine-grain second by second analysis. The setup consisted of a customized plethysmograph for respiration monitoring, equipped with a microphone capturing USV, and with four video cameras for behavior recording. In addition, the bottom of the plethysmograph was equipped with a shock-floor allowing foot-shock delivery, and the top received tubing for odor presentations. Using this experimental setup we first described the characteristics of respiration and USV in different behaviors and emotional states. Then we monitored these parameters during contextual fear conditioning and showed that they bring complementary information about the animal's anxiety state and the strength of aversive memory. The present setup may be valuable in providing a clearer appraisal of the physiological and behavioral changes that occur during acquisition as well as retrieval of emotional memory.

Keywords: respiration, ultrasonic vocalizations, olfaction, emotion, fear, odor-fear conditioning, stress, plethysmograph

\section{INTRODUCTION}

In animals, emotional memory is classically assessed through pavlovian fear conditioning. This paradigm consists of pairing a novel neutral stimulus (the conditioned stimulus) with an aversive unconditioned stimulus, generally a mild foot-shock. After conditioning, the conditioned stimulus elicits a wide range of behavioral and physiological responses that usually occur when an animal encounters a danger (LeDoux, 2000; Maren, 2001). These responses include freezing, potentiated acoustic startle, increased heart rate and blood pressure, breathing modulation, ultrasonic vocalizations (USV), and glucocorticoids release. Despite the existence of this large repertoire of responses, freezing behavior is often the sole parameter measured for quantifying fear response, thus limiting emotional memory appraisal to this unique index. Freezing is characterized by a crouching posture and the absence of any visible movement except that due to breathing (Blanchard and Blanchard, 1969). While freezing is a robust and easily quantifiable response, it lacks temporal sensitivity and plasticity. Indeed, once induced in response to a foot-shock, freezing often persists throughout the session thus precluding the observation of subtle variations in animal's fear levels. Interestingly, respiratory changes and USV can occur during fear response, yet very few studies have addressed the question of the link between these different parameters and freezing and even less studies have correlated them using a fine-grained temporal scale. The reason for this is that no commercial device is available for such an investigation on adult animals.

The aim of the present study was to design an experimental setup allowing both (1) the simultaneous recording of respiration, USV, and behavior (RUB cage) in rats experiencing different emotional states and (2) the offline synchronization of the collected data for fine-grain second by second analysis. The rationale for choosing respiration and USV in addition to freezing is the following. Beside its primary function of maintaining physiological homeostasis, respiration is also modulated by factors which are not primarily related to metabolic requirements, including emotions, arousal, and cognitive activities (Ley, 1999; Gallego and Gaultier, 2000). Emotion is known from a long time to affect respiratory frequency (Stevenson and Ripley, 1952; Boiten, 1998; Homma and Masaoka, 2008). For instance, rats increase their rate of sniffing when exposed to unconditional rewards like rewarding brain stimulation (Rossi and Panksepp, 1992) or reinforcer delivery in motivated animals. In addition, respiration can also be modulated in anticipation of a reward (Clarke and Trowill, 1971; Waranch and Terman, 1975). Increased arousal, induced for example by arrival of an unexpected sensory cue, is another factor known to modulate breathing rhythm. Indeed, sampling a novel odorant (Welker, 1964; 
Macrides et al., 1982; Youngentob et al., 1987; Kepecs et al., 2007; Wesson et al., 2008) or perceiving an auditory or visual stimulus (Freeman et al., 1983; Wesson et al., 2008), results in reliable induction of high-frequency $(6-10 \mathrm{~Hz})$ sniffing. Finally, classical conditioning also plays a significant role in the control of breathing (Freeman et al., 1983; Monod et al., 1989; Nsegbe et al., 1997, 1999), suggesting that sniffing can also occur as a conditioned response when motivationally neutral stimuli are contiguously paired with reinforcing stimuli. In that sense, respiratory activity could be a good index of both novelty detection and emotional learning.

Ultrasonic vocalizations can also bring information in the context of emotional memory. Rats and other rodents have developed communication in the ultrasonic range of sound frequencies. Adult rats emit two types of ultrasonic calls. Twenty-two kilohertz vocalizations are observed in aversive and dangerous situations like predator encounter (Blanchard et al., 1991) or painful stimuli (Borta et al., 2006) and are therefore considered as alarm calls. In contrast, rats emit $50-\mathrm{kHz}$ vocalizations in positive affective states as induced by play (Knutson et al., 1998, 2002), or during sexual interactions (White et al., 1990). Like respiratory activity, USV can be modulated in anticipation of a positive (rewarding brain stimulation: Burgdorf et al., 2000; food anticipation: Knutson et al., 2002) or negative (electric shock: Wohr et al., 2005; Portfors, 2007) outcome. Thus USV, like respiration, could provide complementary information to freezing in the context of emotional learning.

The setup presented here consisted of a customized plethysmograph for respiration monitoring, equipped with a microphone capturing USV, and with video cameras for behavior recording. In addition, the bottom of the plethysmograph was equipped with a shock-floor allowing foot-shock delivery, and the top received tubings for odorant presentations. Offline, the three sets of collected data (respiration, USV, and behavior) were exported to a database where they were synchronized and analyzed. We first characterized the recorded parameters in different emotional states, ranging from low level emotional state (quiet or sleep resulting from exposure to a familiar context), to moderate (increase of arousal induced by a new environment or a novel odor presentation) and high (fear induced by foot-shock delivery) levels. Then, in order to validate this setup in the context of emotional memory, we investigated whether respiration and USV are modulated by contextual fear learning, and can thus be used as reliable indices of anxiety and aversive memory.

\section{MATERIALS AND METHODS ANIMALS}

Data were obtained from 30 male Long-Evans rats (Janvier, France) weighing 250-300 $\mathrm{g}$ at the start of the experimentation. They were housed individually at $23^{\circ} \mathrm{C}$ and maintained under a 12 -h lightdark cycle (lights on from 7:00 a.m. to 7:00 p.m.). Food and water were available ad libitum during the experiment. Experiments were performed in accordance with the European guidelines regarding the care and use of animals for experimental procedures.

\section{EXPERIMENTAL APPARATUS}

The apparatus consisted of a whole body customized plethysmograph (Figure 1A; diameter $20 \mathrm{~cm}$, height $30 \mathrm{~cm}$, EMKA technologies, France; see also Figure A1 in Appendix) placed in a home made sound-attenuating cage ( $\mathrm{L} 60 \mathrm{~cm}, \mathrm{~W} 60 \mathrm{~cm}, \mathrm{H} 70 \mathrm{~cm})$. The plethysmograph was used to measure respiratory parameters in behaving animals. It is composed of two parts which come together to form two independent airtight chambers: the animal and reference chambers. The pressure change due to animal respiration was measured by a differential pressure transducer (Model dpt, EMKA Technologies, France) with one port exposed to the animal chamber and the other to the reference chamber.

The ceiling of the plethysmograph was equipped with a tower which allowed the introduction of three Tygon tubing (diameter $3 \mathrm{~mm}$ ) connected to a programmable homemade olfactometer to deliver air and odorants. Deodorized air flowed constantly through the cage $(2 \mathrm{l} / \mathrm{min})$. When needed, an odor was introduced smoothly in the air stream thus minimizing its effect on change in pressure. The bottom of the animal chamber has a port connected to a ventilation pump which can draw air out of the plethysmograph (at a rate of up to $2 \mathrm{l} / \mathrm{min}$ ) thus maintaining a constant airflow that does not interact with the animal's breathing pattern. A condenser ultrasound microphone (Avisoft-Bioacoustics CM16/CMPA, Berlin, Germany) was inserted in the tower on the top of the plethysmograph to monitor USV emitted by the rats. The bottom of the animal chamber has been equipped with a shock-floor connected to a programmable Coulbourn shocker (Bilaney Consultants GmbH, Düsseldorf, Germany). Animal's behavior was monitored using four video cameras (B/W CMOS PINHOLE camera, Velleman, Belgium) placed at each corner of the sound-attenuating cage. We used an acquisition rate of 25 images per second with a RS170 video format which is a standard black and white video format.

\section{EXPERIMENTAL PARADIGM Experiment I}

This experiment was aimed at characterizing respiration and USV in various behaviors and emotional states. The animals were familiarized to the experimental environment during 4 days (30 min per day) preceding the beginning of the experiments. The rats were then separated in two experimental groups. In the odor group $(n=6)$, the animals were introduced in the experimental cage and allowed a 4-min exploration period after which they received 10 presentations of an odor (20 s duration; Pure Peppermint Extract; McCormick, USA) with a 4-min interval between two presentations. In the shock group $(n=18)$, the rats were introduced in the experimental cage and allowed a 4-min exploration period after which they received 10 foot-shocks $(0.4 \mathrm{~mA}, 1 \mathrm{~s})$ spaced by a 4 -min interval. These conditions allowed us to induce two types of behavioral changes trend: in the odor group, overt behavior changed from exploration of the experimental cage, to active sampling upon new odor arrival, followed by odor habituation and progressive sleep instatement. In the shock group, overt behavior evolved from exploration of the experimental cage, to freezing and USV induction in response to repeated shock delivery. Behavior, respiration, and USV were collected throughout the entire experimental session. Offline, in both groups, we selected periods of the experimental session where the different target behaviors were stable (with a minimum duration of $10 \mathrm{~s}$ ) and unambiguous, and analyzed corresponding respiration and USV. 


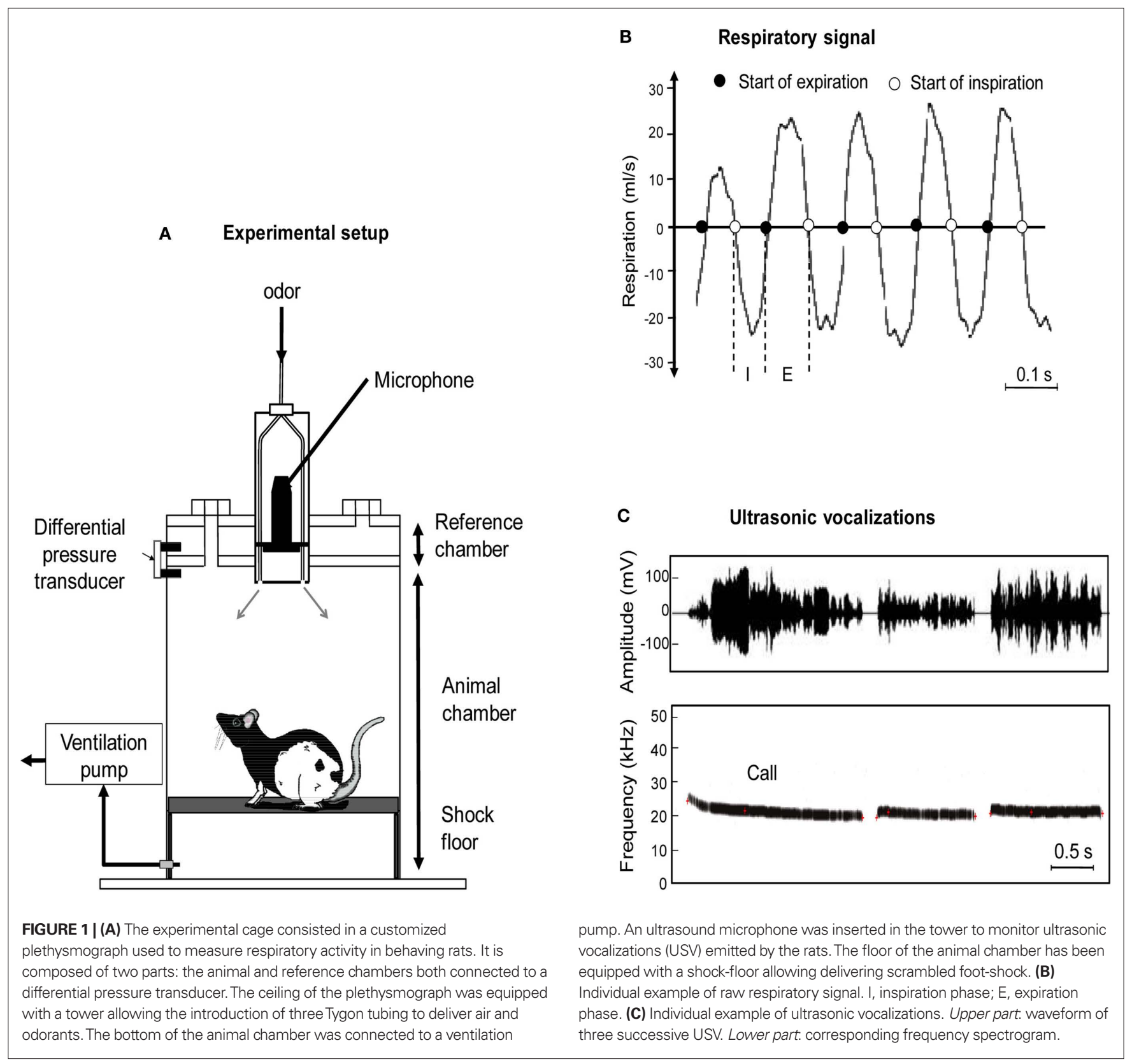

\section{Experiment II}

This experiment was aimed at investigating to what extent respiration and USV are modulated by contextual fear conditioning and can serve as aversive memory indices. Two groups of animals were carried out, based on the intensity of the foot-shock delivered: $0.4 \mathrm{~mA}(n=10)$ or $0.8 \mathrm{~mA}(n=6)$. In each group, the animals were introduced in the experimental cage and allowed a 4-min exploration period after which they received 10 footshocks (1 s) spaced by a 4-min interval. Twenty-four hours later the animals were tested for their retention of the learning. For this, they were reintroduced in the experimental cage for a duration equivalent to the conditioning session. Behavior, respiration, and USV were collected in both groups throughout the acquisition and retention session. Offline, in each group, the different parameters were quantified at three periods of each session: beginning (first foot-shock), middle (after 5 foot-shocks), and end (after 10 foot-shocks).

\section{DATA ACOUISITION AND PRE-PROCESSING Respiration recording}

The respiratory signal collected from the plethysmograph was amplified and sent to an acquisition card (MC-1608FS, Measurement Computing, USA; Sampling rate $=1000 \mathrm{~Hz}$ ) for storage and offline analysis. Using whole body plethysmograph setup, natural breathing signal appears as a periodic phenomenon showing alternating negative (inspiration) and positive (expiration) deflections (Figure 1B). A central point of respiratory signal analysis is the detection of respiratory cycles. In order to do this, we used an algorithm described 
in a previous study (Roux et al., 2006). This algorithm performs two main operations: signal smoothing for noise reduction, and detection of crossing zero points in order to define accurately the inspiration and expiration phase starting points. Inspiration phase started at the zero-crossing point of the respiratory trace falling phase and ended at the zero-crossing point of the respiratory trace rising phase. Expiration phase started at the zero-crossing point of the respiratory trace rising phase and ended at the zero-crossing point of the respiratory trace falling phase (Figure 1B). In addition, in order to eliminate artifacts, we determined a cut-off value for signal duration (rejection if value $<$ median/6) or for signal amplitude (rejection if value $<$ median/3), which allowed us to define minimum duration and amplitude values for a cycle to be accepted as such.

\section{USV recording}

The ultrasound microphone was connected to a recording interface (UltraSoundGate $116 \mathrm{Hb}$, Avisoft-Bioacoustics) with the following settings: sampling rate $=214285 \mathrm{~Hz}$; format $=16$ bit (Wohr et al., 2005). Recordings were transferred to Avisoft SASLab Pro (version 4.2, Avisoft-Bioacoustics, Berlin, Germany) and a fast Fourier transform (FFT) was conducted. Spectrograms were generated with an FFT-length of 512 points and a time window overlap of $87.5 \%$ (100\% Frame, FlatTop window). These parameters produced a spectrogram at a frequency resolution of $419 \mathrm{~Hz}$ and a time resolution of $0.29 \mathrm{~ms}$. The acoustic signal detection was provided by an automatic whistle tracking algorithm with a threshold of $-20 \mathrm{~dB}$, a minimum duration of $0.01 \mathrm{~s}$ and a hold time of $0.02 \mathrm{~s}$. However, the accuracy of detection was verified trial by trial by an experienced user. The main parameters used in the present study were the duration as well as the start and end time of each ultrasonic call (Figure 1C).

\section{Behavior coding}

The video signal collected through the four video cameras was acquired with a homemade acquisition software (Volcan) using the Matrox Imaging Library and a Matrox acquisition card (Morphis QxT 16VD/M4, Matrox Video, UK). Each recording was stored in AVI file format with jpeg codec. Offline, the video recordings were replayed using Volcan software and the animals' behavior was encoded using an ethologic keyboard. Four specific behaviors were encoded: exploration, odor sampling, sleep, and freezing. A specific key on the ethologic keyboard was assigned to each behavior: $\mathrm{e}=$ exploration (ranging from animal displacement to complete exploratory behavior), $\mathrm{o}=$ odor sampling (elevation and active sniffing and whisking with the head oriented upward toward odor source), $\mathrm{s}=$ sleep (eyes closed, body lying on the floor), $\mathrm{f}=$ freezing (crouching posture and absence of any visible movement except that due to breathing; Blanchard and Blanchard, 1969). Using this procedure, animal's behavior encoding resulted in a file containing the time of occurrence and the duration of the target behaviors throughout each session.

\section{DATA SYNCHRONIZATION AND ANALYSIS}

The different data (respiration, USV, and behavior) were then entered in a database OpenElectrophy (previously designed for electrophysiological signals analysis; Garcia and Fourcaud-Trocme,
2009). OpenElectrophy stores experimental data and meta-data in a single central MySQL database, and provides a graphic user interface to visualize and explore the data, and a library of functions for user analysis scripting in Python. OpenElectrophy is open source and is freely available for download at http://neuralensemble.org/trac/OpenElectrophy. The first step of data analysis was data synchronization. This was achieved via a TTL synchronization signal generated at the beginning of each experimental session. Secondary TTL signals were also generated for important events in the session: odor arrival, shock delivery. Once synchronized, the different data stored in the database, could be visualized simultaneously (see Movies S1 and S2 in Supplementary Material). The data were analyzed using analysis scripts in Python. Two strategies were used. First, we correlated the three sets of data 2 by 2: USV versus behavior, respiration versus USV, and behavior versus respiration. (1) USV versus behavior: the behavioral data corresponding to each USV were extracted and their category examined. This allowed us to determine in which behavioral state the USV occurred preferentially. (2) Respiration versus USV: because it rapidly occurred that USV emission has a strong impact on respiration, the respiratory signal corresponding to each USV was extracted and analyzed. Specifically, the duration of expiration and inspiration phases during each call was examined and correlated to the duration of the call. (3) Behavior versus respiration: for each defined behavior (exploration, odor sampling, freezing, and sleep), the corresponding respiratory signals were extracted and their parameters analyzed separately. This allowed us to compare the respiration parameters in the different behaviors. Instantaneous respiratory frequency was first determined as the inverse of the respiratory cycle (inspiration plus expiration) duration. The distribution of respiration frequencies was then examined for each behavioral state, using $0.25-\mathrm{Hz}$ bins.

The second way of analyzing the data was to follow the simultaneous time-course of respiration, USV, and behavior in parallel throughout the experimental session. For this, instant respiratory frequency, animal's behavior duration, and USV duration were averaged on a second by second basis.

\section{STATISTICAL ANALYSIS}

Respiratory frequencies distributions were compared between behaviors using a Kolmogorov-Smirnov non-parametric test. The time-course of mean respiration, behavior, and USV during the session was compared between the experimental groups using a two-way ANOVA for repeated-measures, followed by post hoc Fisher tests comparisons. For all the statistical comparisons performed, the significance level was set at 0.05 .

\section{RESULTS \\ EXPERIMENT I: CHARACTERIZATION OF RESPIRATION AND USV DURING VARIOUS BEHAVIORS AND EMOTIONAL STATES \\ Ultrasonic vocalizations are mainly observed during freezing behavior and drastically affect respiration}

Ten out of the $18(56 \%)$ animals receiving foot-shocks emitted USV. In these animals, we measured the duration of USV emission for different behaviors. We distinguished three main behavioral categories: exploration, freezing, and a third category regrouping the other remaining behaviors (like grooming or sleep). The data 
show that USV are mainly observed during freezing (Figure 2). Indeed, a repeated-measures ANOVA revealed a significant effect of the behavior $[F(2,18)=34.878, p<0.001]$. Post hoc Fisher pair-wise comparisons further showed that the duration of USV emission observed during freezing (expressed as a percentage of behavior duration) was higher than that observed during exploration or other remaining behaviors $(p<0.001)$.

In addition, during USV, the respiratory signal presented a very particular shape (Figure 3A), with a short inspiration preceding call emission and a long expiration concomitant with the vocalization. The duration of USV was then correlated with inspiration and expiration phase duration (Figure 3B). A highly significant correlation was observed between call duration and expiration duration $\left(r^{2}=0.761, p<0.001\right)$, while no significant correlation was found between call duration and inspiration duration.

Ultrasonic vocalizations are therefore essentially observed in stressful situations and their occurrence strongly affects respiration.

\section{Respiratory frequency is characteristic of the animal's behavior}

In order to characterize respiratory frequency in relation to behavior, in the two experimental groups we selected periods of the experimental session where these behaviors were stable (with a minimum duration of $10 \mathrm{~s}$ ) and unambiguous. We focused on four main behaviors: exploration, odor sampling, freezing, and sleep. Exploration was measured in all the rats during the first $4 \mathrm{~min}$ following introduction of the animal in the experimental cage. Odor sampling behavior (odor) was assessed in the odor group, during the first presentation of a novel odor. Freezing behavior and sleep behavior were assessed respectively in shock animals and in odor animals throughout the session. Respiratory signals corresponding to the four behaviors were analyzed separately.

Visual inspection of raw respiratory signals revealed striking differences in signal's shape and frequency in the different behaviors (Figure 4A). Distribution of individual cycle frequency for each behavior is represented in Figure 4B. Statistical analysis showed significant differences between all the distributions (two-sample Kolmogorov-Smirnov comparisons, $p<0.05$ ), with the mean frequency value (insert in Figure 4B) gradually decreasing from odor $(9.1 \pm 0.1 \mathrm{~Hz})$ to exploration $(7.6 \pm 0.1 \mathrm{~Hz})$, freezing $(3.4 \pm 0.2 \mathrm{~Hz})$

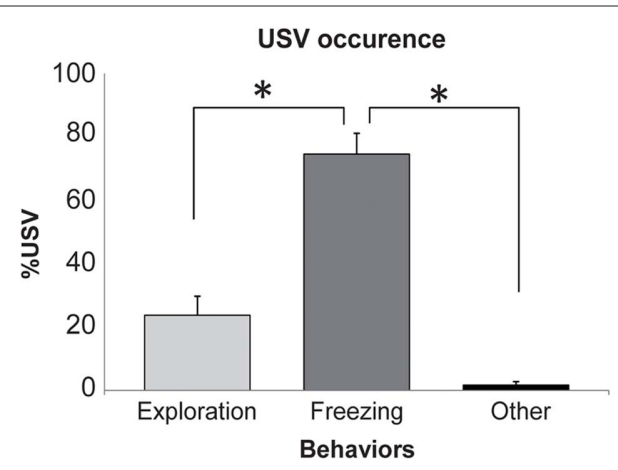

FIGURE 2 | Occurrence of ultrasonic vocalizations (USV) in the different behaviors. For each behavior, the duration of USV was expressed as a percent (mean \pm SEM) of the behavior total duration. * Significant difference $(p>0.001)$. and sleep $(2.0 \pm 0.4 \mathrm{~Hz})$. Pair-wise comparisons revealed that each mean frequency was significantly different from the other three $(p<0.001)$.

A closer examination of frequency distribution curves on Figure 4B shows that the distribution obtained for freezing presents two peaks: the first at $0.5 \mathrm{~Hz}$ and the second at $2.75 \mathrm{~Hz}$. Given that USV emission has a strong impact on respiration and that not all shocked animals produced USV, we pursued the analysis of frequency distribution during freezing on the two groups of animals separately: those emitting USV (USV group, $n=10$ ) and those emitting no USV (no USV group, $n=8$ ). The data showed that the first frequency peak observed during freezing behavior $(0.5 \mathrm{~Hz})$ was due to USV emission

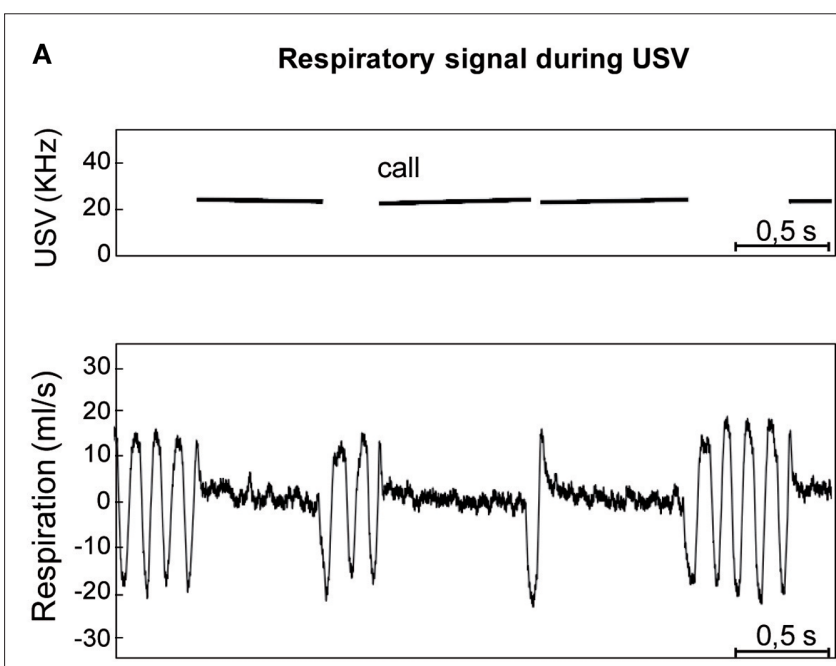

B

Correlation between expiration and call duration

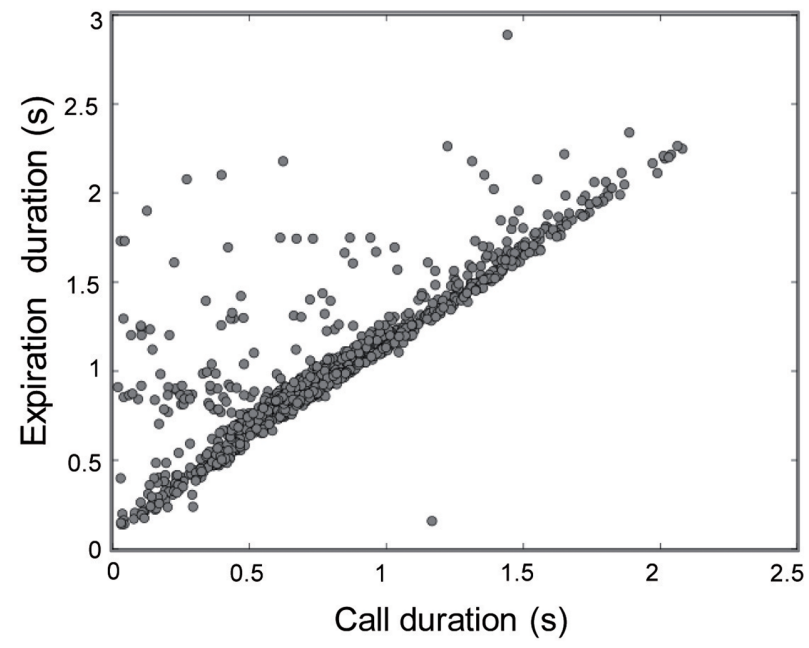

FIGURE 3 | (A) Individual example of the typical respiratory signal observed during ultrasonic vocalizations (USV). Upper part: frequency spectrogram of calls. Lower part: corresponding respiratory signal. For each USV, a short inspiration preceded call emission while a long expiration was concomitant with it. (B) The duration of USV was highly correlated with the duration of the expiration phase $\left(r^{2}=0.761 ; p<0.001\right)$. 


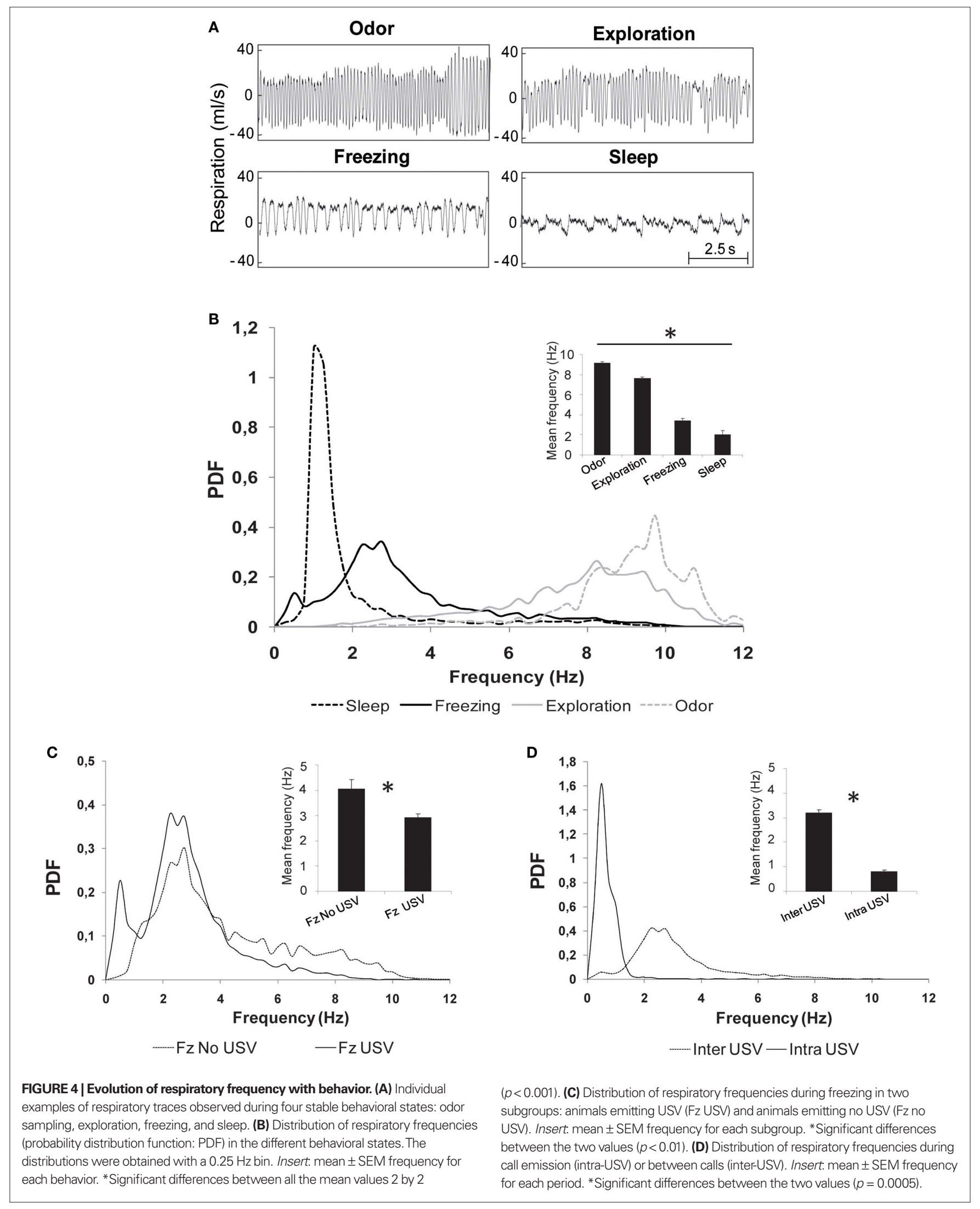


(Figure 4C). Pair-wise comparisons carried out on the mean frequency values in the two groups (insert in Figure 4C) showed that the mean frequency observed in the no USV group $(4.1 \pm 0.4 \mathrm{~Hz})$ was higher than in the USV group $(2.9 \pm 0.1 \mathrm{~Hz} ; p<0.01)$.

In the USV group, periods of call emission were separated by call-free periods. Therefore, we further analyzed the respiratory signal collected during these two periods. The data clearly show different frequency distributions in the two periods (two-sample Kolmogorov-Smirnov comparisons, $p<0.05$ ), with a narrow frequency distribution peaking around $0.5 \mathrm{~Hz}$ during calls emission and a broader distribution peaking around $2.25 \mathrm{~Hz}$ during intercalls periods (Figure 4D). Wilcoxon comparisons carried out on the mean frequency values obtained in the two periods (insert in Figure 4D) showed that the mean frequency during USV (intraUSV, $0.8 \pm 0.05 \mathrm{~Hz}$ ) was lower than that between USV (inter-USV, $3.2 \pm 0.1 \mathrm{~Hz} ; p=0.005)$.

Thus, each target stable behavior studied here can be described by a specific respiration distribution curve and a characteristic mean frequency value.

\section{Respiration is a good index of novel stimulus detection and arousal level}

We investigated whether transient changes could be observed on the respiratory signal when a discrete event, here a new odor $(20 \mathrm{~s}$ duration), was introduced repeatedly (odor group). This paradigm first induced increased arousal due to novel stimulus detection, followed by habituation and progressive instatement of sleep. Since no USV were observed in these conditions, we focused on sleep behavior and respiration. We used a 35-s time window centered on odor presentation, at different periods of the experimental session, i.e., beginning (first odor presentation), middle (after 5 odor presentations), and end (after 10 odor presentations). The data are reported on Figure 5.

Concerning sleep behavior (Figure 5A), a two-way (time, period) repeated-measures ANOVA revealed a significant difference for period $[F(2,15)=11.56, p<0.01]$, time $[F(35,525)=3.71$, $p<0.001]$ and time $\times$ period interaction $[F(70,525)=1.723$, $p<0.001]$. Further comparisons showed that sleep was significantly higher in the middle $(p<0.05)$ and end $(p<0.01)$ of the session than at the beginning.

Concerning respiration (Figure 5B), ANOVA revealed a significant difference for period $[F(2,15)=38.40, p<0.001]$, time $[F(35,525)=9.60, p<0.001]$, and time $\times$ period interaction $[F(70,525)=4.63, p<0.001]$. Further comparisons showed that respiratory frequency was globally significantly higher at the beginning of the session than in the middle $(p<0.01)$ or end $(p<0.001)$. For each period, we then analyzed throughout the 30 s time window, variations in respiratory frequency upon odor introduction, compared to pre-odor levels. Pair-wise comparisons showed that the first presentation of a new odor (beginning period) induced a strong increase in respiratory frequency which reached significant levels $(p<0.05)$ from the 4 th second after odor introduction and lasted until the end of the $30 \mathrm{~s}$ time window. In the middle of the session, presentation of the odor induced a light significant increase in respiratory frequency from second 3 to $11(p<0.05)$ after odor introduction, after which respiration frequency returned to pre-odor baseline levels. At the end of the

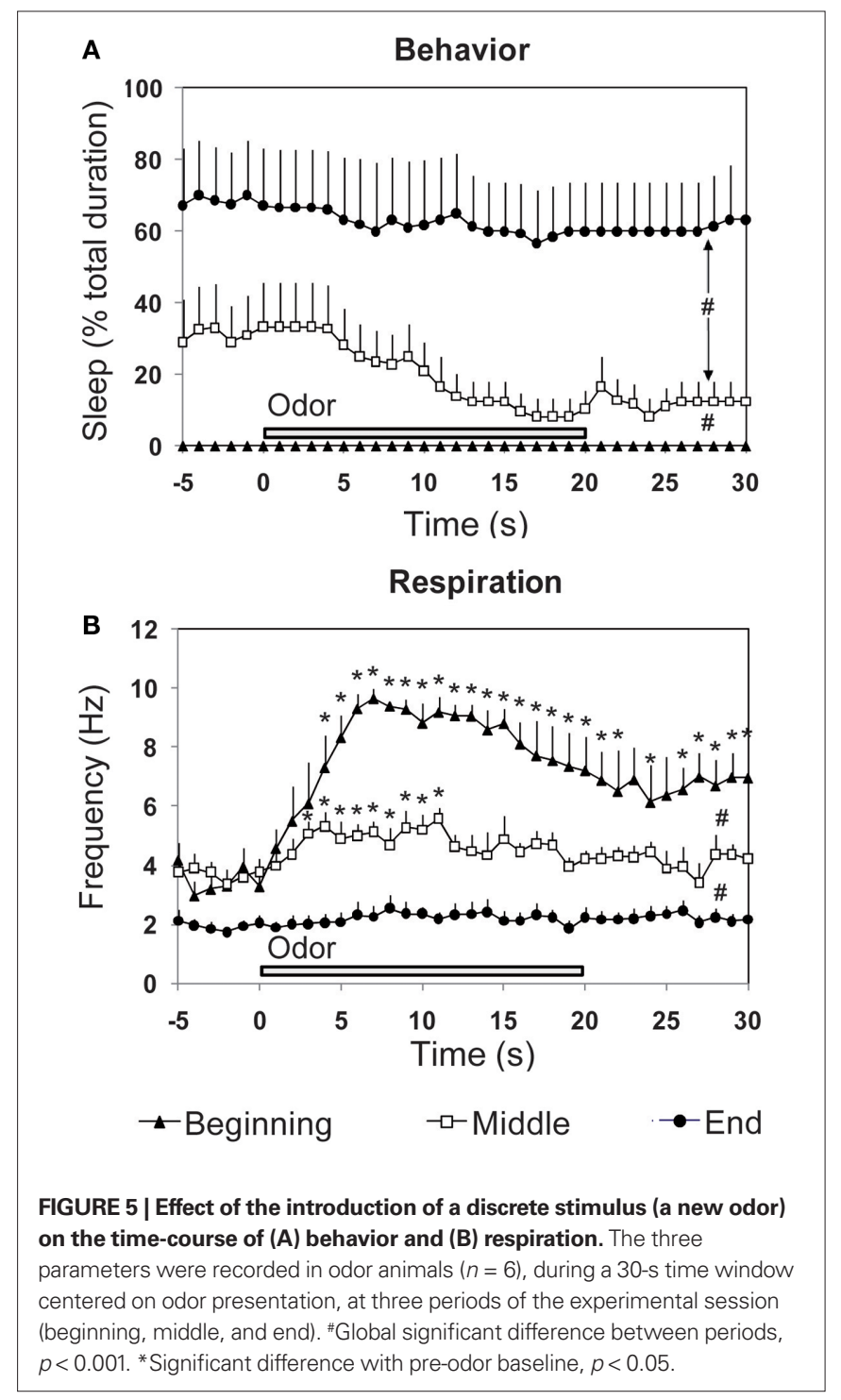

session, pre-odor baseline frequency was significantly lower than that observed for the beginning and middle periods. Furthermore, odor introduction induced no change in frequency.

In summary, introduction of a novel unexpected odor induced a fast and strong increase in respiratory rate. As the odor becomes familiar and its arousing effect decreases, change in respiratory frequency upon odor introduction attenuates to finally disappear, and sleep behavior increases while respiration frequency decreases. Respiration can thus be an accurate index of novel stimulus detection as well as a marker of the animal's global arousal state.

\section{EXPERIMENT II: BEHAVIOR, RESPIRATION, AND USV DURING CONTEXTUAL FEAR CONDITIONING \\ Behavior, respiration, and USV are modulated by the level of stress during acquisition}

Contextual fear conditioning was performed in two groups of rats differing only by the intensity of the applied foot-shock: 0.4 versus $0.8 \mathrm{~mA}$. Behavior, respiration, and USV were monitored in both 
groups during the acquisition session and analyzed at three different periods of the session, i.e., beginning (first foot-shock), middle (after 5 foot-shocks), and end (after 10 foot-shocks).

Concerning freezing behavior, in both groups, high levels of freezing were observed from the middle to the end of the session (Figure 6A, left part). A two-way (group, period) ANOVA for repeated-measures showed a significant effect for group
$[F(1,14)=10.49, p<0.01]$ and for period $[F(2,28)=87.51$, $p<0.0001]$. Further comparisons revealed that $0.8 \mathrm{~mA}$-shock animals displayed higher freezing rate than $0.4 \mathrm{~mA}$-shock animals, for the beginning and end periods $(p<0.05)$. Concerning respiration (Figure 6B, left part), in both groups, respiratory frequency decreased throughout the session. The ANOVA revealed a significant effect for group $[F(1,14)=20.86, p<0.005]$ and

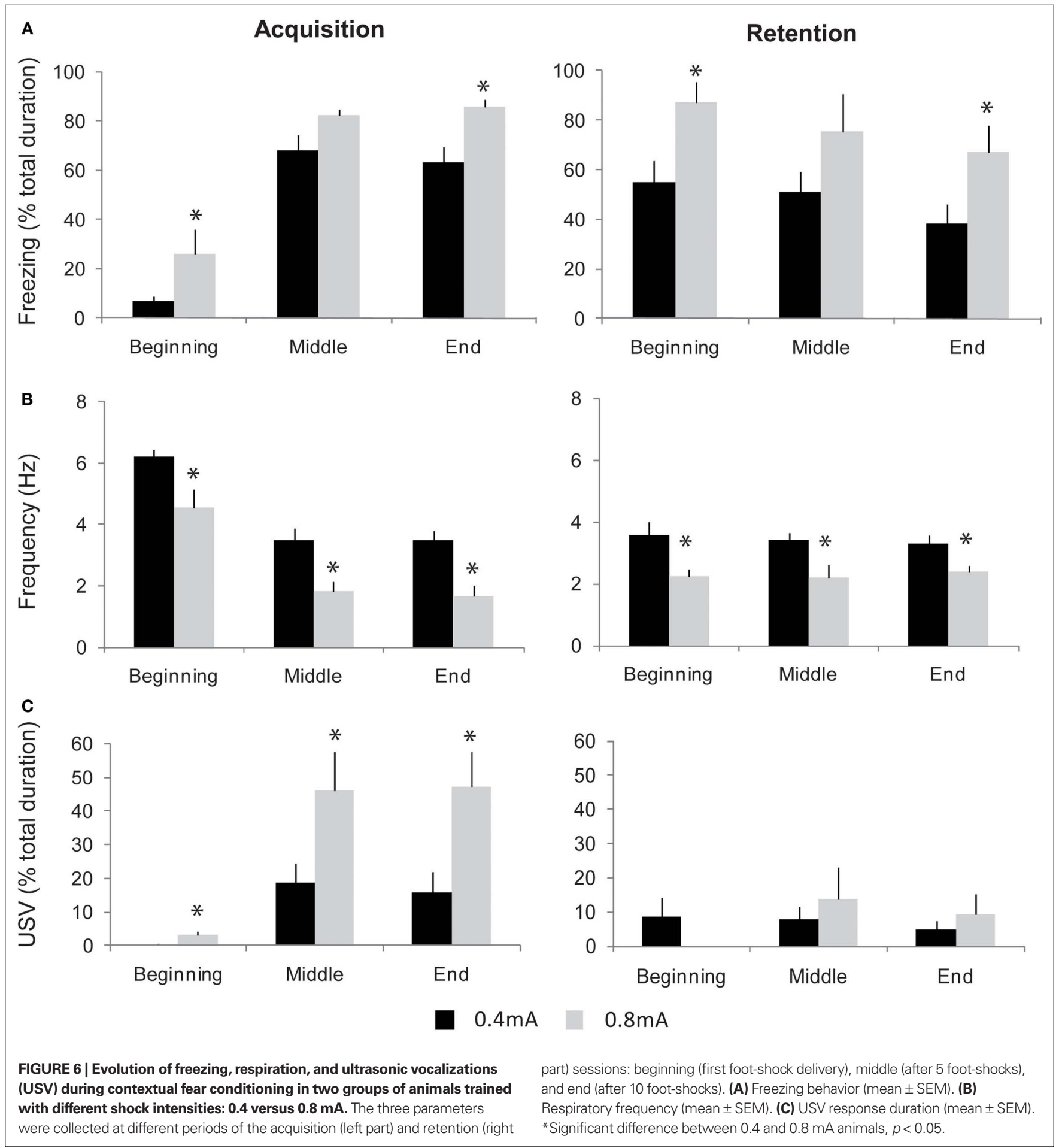


period $[F(2,28)=50.02, p<0.0001]$. Further pair-wise comparisons showed that $0.8 \mathrm{~mA}$-shock animals displayed a lower respiratory frequency than $0.4 \mathrm{~mA}$-shock animals, for the beginning, middle, and end periods $(p<0.05)$. Concerning USV, 50\% $(5 / 10)$ of the rats in the $0.4 \mathrm{~mA}$-shock group emitted USV in response to shock delivery, against $100 \%(6 / 6$; Chi-square test, $p=0.027)$ in the $0.8 \mathrm{~mA}$-shock group. Additionally when considering the duration of USV emission during the session in both groups (Figure 6C, left part), the ANOVA revealed a main effect of group $[F(1,14)=7.07$, $p<0.05]$ and period $[F(2,28)=27.56, p<0.05]$. Post hoc comparisons showed that $0.8 \mathrm{~mA}$-shock animals displayed longer duration USV emission than $0.4 \mathrm{~mA}$-shock animals, all along the session $(p<0.05)$. The increase in USV duration was mainly due to the fact that the average number of calls per rat was higher in $0.8 \mathrm{~mA}$-shock rats $(1518 \pm 359)$ than in $0.4 \mathrm{~mA}$-shock animals $(620 \pm 126 ; p<0.05)$.

In summary, in animals receiving repeated foot-shocks, a global state of fear builds up during the session, characterized in all animals, by an increase in freezing amount together with a decrease in respiratory frequency, and in a subset of animals, by the emission of USV. Increasing unconditioned stimulus (foot-shock) intensity induced a further enhancement of freezing and USV response as well as a greater decrease in respiratory rate.

\section{Respiration and behavior are good indices of aversive emotional memory}

Twenty-four hours after acquisition, respiration, USV, and freezing were monitored in the two groups when re-exposed to the context during a retention session. Concerning freezing (Figure 6A, right part), both groups exhibited high levels of freezing throughout the session, thus showing the animals have developed a conditioned fear to the context. A two-way (group, period) repeatedmeasures ANOVA showed an effect for group $[F(1,14)=5.51$, $p<0.05]$ and period $[F(2,28)=4.11, p<0.05]$. Further comparisons revealed that the freezing rate was higher in $0.8 \mathrm{~mA}$-shock animals than in $0.4 \mathrm{~mA}$-shock animals, for the beginning and end periods $(p<0.05)$. Concerning respiration, in both groups from the beginning of the session, the respiratory rate was lower than that observed before conditioning (Figure 6B, right part). The ANOVA revealed a significant effect of group $[F(1,14)=15.34$, $p<0.005$ ] with no effect for period. Further pair-wise comparisons showed that $0.8 \mathrm{~mA}$-shock animals displayed a lower respiratory frequency than $0.4 \mathrm{~mA}$-shock animals throughout the retention session $(p<0.05)$. Regarding USV, the percentage of rats emitting USV was lower than during conditioning and similar in both groups (4/10, i.e., $40 \%$ in $0.4 \mathrm{~mA}$-shock group versus $2 / 6$, i.e., $33 \%$ in $0.8 \mathrm{~mA}$-shock group), as was the global duration of USV emission (Figure 6C, right part).

Thus, the fear conditioning paradigm used here resulted in a conditioned contextual freezing response during the retention session, together with a decrease in respiratory rate. This effect was dose-dependent since increasing shock intensity during acquisition resulted in an enhancement of the conditioned behavioral and respiratory responses observed during retention. In contrast, the likelihood of USV response was lower during retention than during conditioning, and did not vary with shock intensity.

\section{DISCUSSION}

The present study was devoted to design and validate an experimental setup allowing recording three parameters in parallel (the RUB cage), in animals experiencing different emotional states. To our knowledge, the present setup is the first to afford such possibilities in adult behaving rats. Using this experimental setup, in Experiment I we first studied the characteristics of respiration and USV in different behaviors and emotional states. We showed that respiration is affected by the animal's behavior, and each target behavior studied here could be described by a specific respiration distribution curve and a characteristic mean frequency value. Respiration also proved to be an accurate index of stimulus novelty detection and of transient changes in arousal state. USV are essentially observed in stressful situations and their occurrence strongly affects respiration. In Experiment II, we monitored respiration, USV, and behavior during contextual fear conditioning using two different foot-shock intensities. We showed that respiration and USV are differentially modulated by learning and therefore constitute complementary indices of animal's anxiety state and aversive memory.

\section{DIFFERENT BEHAVIORS ARE CHARACTERIZED BY SPECIFIC RESPIRATORY RHYTHMS}

In Experiment I, using two experimental paradigms (odor group versus shock group), we induced stable behaviors with emotional levels ranging from low (exposure to a familiar environment) to medium (exposure to a new environment, or a novel odor) and high (repeated foot-shock delivery) levels. This allowed us to screen four main behaviors: odor sampling, exploration, freezing, and sleep. We showed that each behavior was characterized by a specific respiratory frequency distribution curve and mean frequency value.

Low emotional level as obtained during sleep resulted in the lowest respiratory frequency, around $2 \mathrm{~Hz}$. Moderate emotional level observed when the animal was introduced in the experimental cage and engaged in exploratory behavior, led to high respiratory rate $(7.6 \mathrm{~Hz})$. When the level of arousal was further increased by introduction of a novel odor, the respiratory frequency was significantly enhanced $(9.1 \mathrm{~Hz})$. This observation is in accordance with previous reports (Welker, 1964; Macrides et al., 1982; Youngentob et al., 1987; Kepecs et al., 2007; Wesson et al., 2008), and brings further support to the idea that sniffing behavior is functionally important in facilitating odor detection and identification (Youngentob et al., 1987; Buonviso et al., 2006; Schoenfeld and Cleland, 2006; Rennaker et al., 2007; Verhagen et al., 2007; Courtiol et al., 2011). Finally when animals are experiencing fear and exhibit freezing behavior, their respiratory rate is low $(3.4 \mathrm{~Hz})$. Thus, as previously described by Frysztak and Neafsey (1991), a negative affective state as that induced by repeated aversive footshocks, slows down respiratory rhythm. In contrast, positive affective states, like those related to rewarding brain stimulation or food reward anticipation, have been shown to increase sniffing rate (Knutson et al., 2002). For instance, Bindra and Campbell (1967) reported that after pairing a tone with rewarding stimulation of the hypothalamus, the tone came to elicit walking and rearing while sniffing in rats. Similarly, different studies have shown that when a neutral stimulus is paired with a reward in a classical conditioning paradigm, high-frequency sniffing develops in 
anticipation of reward delivery (Freeman et al., 1983; Kepecs et al., 2007; Wesson et al., 2008). Interestingly, Kepecs et al. (2007) using an odor discrimination task allowing to separate odor-sampling and reward-anticipation periods, showed that reward-anticipatory sniffing occurred in a higher-frequency range $(9-12 \mathrm{~Hz})$ compared with odor sampling sniffing $(6-9 \mathrm{~Hz})$.

We also investigated the time-course of respiration upon introduction of a new unexpected odor. We observed that a new odor induced a clear-cut increase in animal's sniffing rate, which reached significant levels $4 \mathrm{~s}$ on average after stimulus onset. This delay likely reflected the time necessary for the odorant to diffuse throughout the chamber and reach the animal's nostrils. Odors are not the unique stimuli that can modulate respiration. Indeed unexpected auditory as well as visual stimuli have previously been shown to induce sniffing behavior (Welker, 1964; Freeman et al., 1983; Wesson et al., 2008). Respiration can therefore be used as a reliable and precise index of animal's increased arousal due to perception of an unexpected event.

Taken together these data suggest that monitoring respiration in learning paradigms can help to gain insight in animal's motivational drives and emotional states.

\section{USV ARE ESSENTIALLY OBSERVED IN STRESSFUL SITUATIONS}

Data from Experiment I showed that USV are produced essentially during high anxiety states characterized by freezing, and are not induced by exposure to a novel odor or a new context. The link between USV and freezing has been reported in previous studies showing that while immobility may occur without $22-\mathrm{kHz}$ USV, such USV do not occur without immobility (Brudzynski and Ociepa, 1992; Walker and Carrive, 2003; Wohr et al., 2005). USV are vibrations caused when expired air is forced through a constricted larynx. Walker and Carrive (2003) suggested that USV may be the consequence of a thorax immobilization procedure, itself a component of the freezing posture whose role is to stabilize both the lower and upper parts of the trunk. This posture may allow a better use of the four limbs for an adapted optimal response to a danger.

\section{RESPIRATION AND ULTRASONIC VOCALIZATIONS: A TIGHT INTERACTION}

Simultaneous recording of respiration and USV allowed us to show that the presence of USV $(22 \mathrm{kHz})$ during freezing induced a lowering in respiratory frequency, and this was due to the drastic lengthening of expiration during call emission. Indeed, during USV, rats took a quick inspiration followed by a prolonged expiration associated with call emission. This resulted in a strong correlation between expiration duration and USV duration. Previous studies reported that USV during immobility occurs when the animal exhales deeply, which is paralleled by increased blood pressure and heart rate (Frysztak and Neafsey, 1991; Antoniadis and McDonald, 1999; Walker and Carrive, 2003). The present experimental setup allowed a precise quantification of the link between $22-\mathrm{kHz}$ USV emission and respiration. In addition, it also provided the opportunity to analyze separately freezing including USV versus USV-free freezing. We observed that although the respiration rate during USV-free freezing is higher than during USV emission, it is nevertheless lower than during exploration or odor sampling, thus showing that in our experimental conditions, a global state of fear or anxiety is associated with a decrease in respiratory rhythm.

\section{RESPIRATION AND 22-kHz USV ARE GOOD INDICES OF ANXIETY LEVELS AND AVERSIVE MEMORY}

In order to validate the present setup in the context of emotional memory, in Experiment II we investigated in which way an aversive learning modulated respiration and USV. For this, we carried out a contextual fear conditioning procedure using two foot-shock intensities: 0.4 versus $0.8 \mathrm{~mA}$, since previous studies have reported a proportional relationship between shock intensity and conditioned freezing response to the context (reviewed in Luyten et al., 2011). During acquisition, in both groups, repeated foot-shocks induced an increase in freezing amount together with a decrease in respiratory rhythm, and the emergence of USV. For each parameter, the effects were greater for the high foot-shock intensity than for the low intensity, therefore suggesting that USV and respiration are reliable indices of the animal's level of stress. Concerning USV, only $50 \%$ of the rats in the $0.4 \mathrm{~mA}$ group emitted USV. This is in accordance with previous observations showing that the likelihood of USV emission in response to aversive stimuli can differ considerably between subjects and that such variability can be due to individual levels of anxietyrelated behavior. Indeed, more anxious rats (as assessed through the elevated plus maze) were shown to exhibit more freezing and to be more likely to vocalize than less anxious animals (Borta et al., 2006). In the present study increasing foot-shock intensity enhanced USV emission. Indeed $100 \%$ animals of the $0.8 \mathrm{~mA}$ group exhibited USV. Moreover the average number of calls per animal was greater in this group. This observation confirms previous data showing that the likelihood of USV occurrence (i.e., call rate, number of rats vocalizing) increased with increasing shock intensity (Wohr et al., 2005). Concerning respiration, the stress-induced decrease in respiratory rhythm was greater in $0.8 \mathrm{~mA}$ than in $0.4 \mathrm{~mA}$ group. Due to the strong constraint imposed by USV emission on respiratory rhythm, the increase in USV observed in $0.8 \mathrm{~mA}$ group might be responsible for the additional decrease in respiratory frequency in this group.

Freezing, respiration, and USV were also measured in the two groups during a retention session carried out $24 \mathrm{~h}$ after acquisition. Both shock intensities produced reliable contextual conditioning as attested by the high level of freezing during retention. Moreover in $0.8 \mathrm{~mA}$ group, freezing was higher than in $0.4 \mathrm{~mA}$ group. In parallel, respiratory frequency was lower in $0.8 \mathrm{~mA}$ group than in $0.4 \mathrm{~mA}$. Thus respiratory response can be conditioned in a dose-dependent manner and therefore represents a good index of the strength of aversive memory. In contrast in both groups, USV emission during the retention session was low and not significantly altered by conditioning shock intensity. This is in accordance with previous data showing that conditioned vocalizations to the context are either absent (Wohr et al., 2005), or necessitate several conditioning sessions to develop (Antoniadis and McDonald, 1999). Thus beside its obvious role as an anxiety index, USV cannot be taken as a reliable aversive memory index, at least in contextual fear conditioning. Interestingly, Wohr et al. (2005) reported that a tone-shock fear conditioning paradigm was efficient to induce acute and conditioned responses in USV. Therefore USV might be a better index of aversive memory in cued rather than context fear conditioning. 


\section{CONCLUSION AND PERSPECTIVES}

The present setup may be valuable in providing a clearer appraisal of the physiological and behavioral changes that occur during acquisition as well as retrieval of emotional memory. Indeed, respiration and USV bring complementary information to behavior. Our data show that respiration is a highly flexible parameter that can both constitute an accurate marker of the animal's sensory detection of a novel stimulus and can also be used as an index of the strength of an aversive memory. USV appear to more specifically reflect an acute level of stress linked to a repeated aversive event, and might also prove useful in identifying animals with different anxiety traits. Therefore respiration and USV can bring useful information at the different critical steps of a learning task.

As illustrated here, this setup might be particularly useful in fear conditioning paradigms. Indeed, a few studies have shown that respiration (Freeman et al., 1983; Monod et al., 1989; Nsegbe et al., 1997, 1999; Wesson et al., 2008) and USV (Burgdorf et al., 2000; Knutson et al., 2002; Wohr et al., 2005; Portfors, 2007) are modulated by classical conditioning and can take part in the conditional response. Using the present setup would allow to follow these parameters in the same animals throughout conditioning thus increasing the sensitivity of the assessment of the fear response. For example in odor-fear conditioning procedure, pairing an odor with foot-shock has been shown to induce high levels of freezing which, once instated, lasted until the end of the acquisition session (Sevelinges et al., 2004; Hegoburu et al., 2009). Thus behavior alone cannot provide clear-cut information about the progressive learning of the odor during the acquisition session since the animal is freezing all the time. Respiration in contrast is a more phasic signal than freezing and could give access to transient changes due to odor arrival, otherwise overshadowed by constant freezing.

Like respiratory activity, USV can be modulated in anticipation of a positive (Burgdorf et al., 2000; Knutson et al., 2002) or negative (Wohr et al., 2005; Portfors, 2007) outcome. Thus USV, like respiration, can provide additional information to freezing behavior in the context of emotional learning. In particular, USV were found useful for differentiating fear and anxiety in rats (Jelen et al., 2003). Indeed in a classical conditioning paradigm, danger stimulus, preceding unavoidable tail shock, is known to induce acute fear, whereas intertrial period evokes anxiety. Interestingly, while freezing rate is often similar in fear and anxiety states, sustained $22-\mathrm{kHz}$ USV were shown to occur preferentially between trials (and may thus characterize anxiety) whereas acute fear induced by a signal of danger resulted in immediate inhibition of USV (Frysztak and Neafsey, 1991; Jelen et al., 2003).

\section{REFERENCES}

Antoniadis, E. A., and McDonald, R. J. (1999). Discriminative fear conditioning to context expressed by multiple measures of fear in the rat. Behav. Brain Res. 101, 1-13.

Bindra, D., and Campbell, J. F. (1967). Motivational effects of rewarding intracranial stimulation. Nature 215, 375-376.

Blanchard, R. J., and Blanchard, D. C. (1969). Crouching as an index of fear. J. Comp. Physiol. Psychol.67, 370-375.

The present setup may also be of great interest for evaluating emotional memory in pups. Indeed in rat pups, odor-shock learning has been shown to occur at early postnatal developmental ages (Sullivan, 2001; Sullivan and Holman, 2010) and to also modulate emotionality and cognitive abilities at adulthood (Sevelinges et al., $2007,2008,2011)$. While typical adult-like freezing response only occurs around postnatal day 15 , sniffing activity as well as USV, are present at the earliest stages of development (Welker, 1964; Hofer et al., 2002; Ehret, 2005; Portfors, 2007). Therefore, monitoring respiration and USV in addition to behavior, gives the opportunity to investigate with the same physiological indices, the development of emotional memory from early postnatal ages until adulthood.

Finally the present experimental apparatus could be further upgraded by adding electrophysiological recordings in target structures using wireless device (Szuts et al., 2011). Such a setup would allow for example to monitor amygdala and olfactory cortex activity, two structures suspected to be involved in odor-fear conditioning (Otto et al., 2000; Sevelinges et al., 2004; Li et al., 2008; Hegoburu et al., 2009), and correlate these activities with the precise time-course of the other recorded parameters.

\section{ACKNOWLEDGMENTS}

This work was supported by the ANR-07-NEURO-O48 MEMOTIME grant to Kiseko Shionoya and Anne-Marie Mouly, by a CNRS PICS Program funding to Chloé Hegoburu and Anne-Marie Mouly, and by a PUF funding to Chloé Hegoburu and Anne-Marie Mouly.

\section{SUPPLEMENTARY MATERIAL}

The Movies 1 and 2 for this article can be found online at http://www.frontiersin.org/behavioral_neuroscience/10.3389/ fnbeh.2011.00025/abstract

\section{Movie S1 | Offline replay of the recorded parameters after their} synchronization. This example was taken from a rat of the shock group, emitting ultrasonic vocalizations (USV). Left part: images collected from the four video cameras. Upper right part. frequency $(\mathrm{kHz})$ spectrogram of the USV across time. Lower right part. respiratory raw activity $(\mathrm{ml} / \mathrm{s})$ across time. The behavioral state corresponding to the ongoing USV content and respiratory activity is symbolized by the colored line under the respiratory signal. Each color represents a given behavior. The vertical red cursor materializes the time corresponding to the video images.

Movie S2 | Offline replay of the recorded parameters after their synchronization. This example was taken from a rat of the odor group. Left part. images collected from the four video cameras. Upper right part: frequency $(\mathrm{kHz})$ spectrogram of the ultrasonic vocalizations (USV) across time. In this group, no USV were observed. Lower right part. respiratory raw activity $(\mathrm{ml} / \mathrm{s})$ across time. The behavioral state corresponding to the ongoing USV content and respiratory activity is symbolized by the colored line under the respiratory signal. Each color represents a given behavior The vertical red cursor materializes the time corresponding to the video images.

anxiety-related behavior. Behav. Brain Res. 166, 271-280.

Brudzynski, S. M., and Ociepa, D. (1992). Ultrasonic vocalization of laboratory rats in response to handling and touch. Physiol. Behav. 52, 655-660.

Buonviso, N., Amat, C., and Litaudon, P. (2006). Respiratory modulation of olfactory neurons in the rodent brain. Chem. Senses 31, 145-154.

Burgdorf, J., Knutson, B., and Panksepp, J. (2000). Anticipation of rewarding electrical brain stimulation evokes ultrasonic vocalization in rats. Behav. Neurosci. 114, 320-327.

Clarke, S., and Trowill, J. A. (1971). Sniffing and motivated behavior in the rat. Physiol. Behav. 6, 49-52.

Courtiol, E., Amat, C., Thevenet, M., Messaoudi, B., Garcia, S., and Buonviso, N. (2011). Reshaping of bulbar odor response by nasal flow rate in the rat. PLOS ONE 6, e16445. doi: 10.1371/journal.pone.0016445

Ehret, G. (2005). Infant rodent ultrasounds - a gate to the understanding 
of sound communication. Behav. Genet. 35, 19-29.

Freeman, W. J., Viana Di Prisco, G., Davis, G. W., and Whitney, T. M. (1983). Conditioning of relative frequency of sniffing by rabbits to odors. J. Comp. Psychol. 97, 12-23.

Frysztak, R. J., and Neafsey, E. J. (1991). The effect of medial frontal cortex lesions on respiration, "freezing," and ultrasonic vocalizations during conditioned emotional responses in rats. Cereb. Cortex 1, 418-425.

Gallego, J., and Gaultier, C. (2000). Respiratory behavior. Rev. Mal. Respir. $17,41-49$.

Garcia, S., and Fourcaud-Trocme, N. (2009). OpenElectrophy: an electrophysiological data- and analysis-sharing framework. Front. Neuroinformatics 3:14. doi: 10.3389/ neuro.11.014.2009

Hegoburu, C., Sevelinges, Y., Thevenet, M., Gervais, R., Parrot, S., and Mouly, A. M. (2009). Differential dynamics of amino acid release in the amygdala and olfactory cortex during odor fear acquisition as revealed with simultaneous high temporal resolution microdialysis. Learn. Mem. 16, 687-697.

Hofer, M. A., Shair, H. N., and Brunelli, S. A. (2002). Ultrasonic vocalizations in rat and mouse pups. Curr. Protoc. Neurosci. Chapter 8:Unit 8.14.

Homma, I., and Masaoka, Y. (2008). Breathing rhythms and emotions. Exp. Physiol. 93, 1011-1021.

Jelen, P., Soltysik, S., and Zagrodzka, J. (2003).22-kHz ultrasonic vocalization in rats as an index of anxiety but not fear: behavioral and pharmacological modulation of affective state. Behav. Brain Res. 141, 63-72.

Kepecs, A., Uchida, N., and Mainen, Z. F. (2007). Rapid and precise control of sniffing during olfactory discrimination in rats. J. Neurophysiol. 98, 205-213.

Knutson, B., Burgdorf, J., and Panksepp, J. (1998). Anticipation of play elicits high-frequency ultrasonic vocalizations in young rats. J. Comp. Psychol. 112, 65-73.

Knutson, B., Burgdorf, J., and Panksepp, J. (2002). Ultrasonic vocalizations as indices of affective states in rats. Psychol. Bull. 128, 961-977.

LeDoux, J. E. (2000). Emotion circuits in the brain. Annu. Rev. Neurosci. 23, 155-184.

Ley, R. (1999). The modification of breathing behavior. Pavlovian and operant control in emotion and cognition. Behav. Modif. 23, 441-479.

Li, W., Howard, J. D., Parrish, T. B., and Gottfried, J. A. (2008). Aversive learning enhances perceptual and cortical discrimination of indiscriminable odor cues. Science 319, 1842-1845.

Luyten, L., Vansteenwegen, D., van Kuyck, K., Deckers, D., and Nuttin, B. (2011). Optimization of a contextual conditioning protocol for rats using combined measurements of startle amplitude and freezing: the effects of shock intensity and different types of conditioning. J. Neurosci. Methods 194, 305-311.

Macrides, F., Eichenbaum, H. B., and Forbes, W. B. (1982). Temporal relationship between sniffing and the limbic theta rhythm during odor discrimination reversal learning. J. Neurosci. 2, 1705-1717.

Maren, S. (2001). Neurobiology of Pavlovian fear conditioning. Annu. Rev. Neurosci. 24, 897-931.

Monod, B., Mouly, A. M., Vigouroux, M., and Holley, A. (1989). An investigation of some temporal aspects of olfactory coding with the model of multi-site electrical stimulation of the olfactory bulb in the rat. Behav. Brain Res. 33, 51-63.

Nsegbe, E., Vardon, G., Perruchet, P., and Gallego, J. (1997).Classic conditioning of the ventilatory responses in rats. $J$. Appl. Physiol. 83, 1174-1183.

Nsegbe, E., Villaret, E., Renolleau, S., Vardon, G., Gaultier, C., and Gallego, J. (1999). Behavioural correlates of conditioned ventilatory responses to hypoxia in rats. Behav. Brain Res. 106, 29-37.

Otto, T., Cousens, G., and Herzog, C. (2000). Behavioral and neuropsychological foundations of olfactory fear conditioning. Behav. Brain Res. $110,119-128$.

Portfors, C.V. (2007). Types and functions of ultrasonic vocalizations in laboratory rats and mice. J. Am. Assoc. Lab. Anim. Sci. 46, 28-34.

Rennaker, R. L., Chen, C. F., Ruyle, A. M., Sloan, A. M., and Wilson, D. A. (2007). Spatial and temporal distribution of odorant-evoked activity in the piriform cortex. J. Neurosci. 27, 1534-1542.

Rossi, J. III, and Panksepp, J. (1992). Analysis of the relationships between self-stimulation sniffing and brainstimulation sniffing. Physiol. Behav. 51, 805-813.

Roux, S. G., Garcia, S., Bertrand, B., Cenier, T., Vigouroux, M., Buonviso, N., and
Litaudon, P. (2006). Respiratory cycle as time basis: an improved method for averaging olfactory neural events. J. Neurosci. Methods 152, 173-178.

Schoenfeld, T. A., and Cleland, T. A. (2006). Anatomical contributions to odorant sampling and representation in rodents: zoning in on sniffing behavior. Chem. Senses 31, 131-144.

Sevelinges, Y., Gervais, R., Messaoudi, B., Granjon, L., and Mouly, A. M. (2004). Olfactory fear conditioning induces field potential potentiation in rat olfactory cortex and amygdala. Learn. Mem. 11, 761-769.

Sevelinges, Y., Moriceau, S., Holman, P., Miner, C., Muzny, K., Gervais, R., Mouly, A. M., and Sullivan, R. M. (2007). Enduring effects of infant memories: infant odor-shock conditioning attenuates amygdala activity and adult fear conditioning. Biol. Psychiatry 62, 1070-1079.

Sevelinges, Y., Mouly, A. M., Raineki, C., Moriceau, S., Forest, C., and Sullivan, R. M. (2011). Adult depression-like behavior, amygdala and olfactory cortex functions are restored by odor previously paired with shock during infant's sensitive period attachment learning. Dev. Cogn. Neurosci. 1,77-87.

Sevelinges, Y., Sullivan, R. M., Messaoudi, B., and Mouly, A. M. (2008). Neonatal odor-shock conditioning alters the neural network involved in odor fear learning at adulthood. Learn. Mem. $15,649-656$.

Stevenson, I., and Ripley, H. S. (1952). Variations in respiration and in respiratory symptoms during changes in emotion. Psychosom. Med. 14, 476-490.

Sullivan, R. M. (2001). Unique characteristics of neonatal classical conditioning: the role of the amygdala and locus coeruleus. Integr. Physiol. Behav. Sci. 36, 293-307.

Sullivan, R. M., and Holman, P. J. (2010). Transitions in sensitive period attachment learning in infancy: the role of corticosterone. Neurosci. Biobehav. Rev. 34, 835-844.

Szuts, T. A., Fadeyev, V., Kachiguine, S., Sher, A., Grivich, M. V., Agrochao, M., Hottowy, P., Dabrowski, W., Lubenov, E. V., Siapas, A. G., Uchida, N., Litke, A. M., and Meister, M. (2011). A wireless multi-channel neural amplifier for freely moving animals. Nat. Neurosci. 14, 263-269.

Verhagen, J. V., Wesson, D. W., Netoff, T. I., White, J. A., and Wachowiak, M. (2007). Sniffing controls an adaptive filter of sensory input to the olfactory bulb. Nat. Neurosci. 10, 631-639.

Walker, P., and Carrive, P. (2003). Role of ventrolateral periaqueductal gray neurons in the behavioral and cardiovascular responses to contextual conditioned fear and poststress recovery. Neuroscience 116, 897-912.

Waranch, H. R., and Terman, M. (1975). Control of the rat's sniffing behavior by response-independent and dependent schedules of reinforcing brain stimulation. Physiol. Behav. 15, 365-372.

Welker, W. I. (1964). Analysis of sniffing in the albino rat. Behavior 22, 223-244.

Wesson, D. W., Donahou, T. N., Johnson, M. O., and Wachowiak, M. (2008). Sniffing behavior of mice during performance in odor-guided tasks. Chem. Senses 33, 581-596.

White, N. R., Cagiano, R., Moises, A. U. and Barfield, R. J. (1990). Changes in mating vocalizations over the ejaculatory series in rats (Rattus norvegicus). J. Comp. Psychol. 104, 255-262.

Wohr, M., Borta, A., and Schwarting, R. K. (2005). Overt behavior and ultrasonic vocalization in a fear conditioning paradigm: a dose-response study in the rat. Neurobiol. Learn. Mem. 84, 228-240.

Youngentob, S. L., Mozell, M. M., Sheehe, P. R., and Hornung, D. E. (1987). A quantitative analysis of sniffing strategies in rats performing odor detection tasks. Physiol. Behav. 41, 59-69.

Conflict of Interest Statement: The authors declare that the research was conducted in the absence of any commercial or financial relationships that could be construed as a potential conflict of interest.

Received: 15 February 2011; accepted: 05 May 2011; published online: 19 May 2011. Citation: Hegoburu C, Shionoya K, Garcia $S$, Messaoudi B, Thévenet $M$ and Mouly A-M (2011) The RUB cage: respirationultrasonic vocalizations-behavior acquisition setup for assessing emotional memory in rats. Front. Behav. Neurosci. 5:25. doi 10.3389/fnbeh.2011.00025

Copyright @ 2011 Hegoburu, Shionoya, Garcia, Messaoudi, Thévenet and Mouly. This is an open-access article subject to a non-exclusive license between the authors and Frontiers Media SA, which permits use, distribution and reproduction in other forums, provided the original authors and source are credited and other Frontiers conditions are complied with. 


\section{APPENDIX}

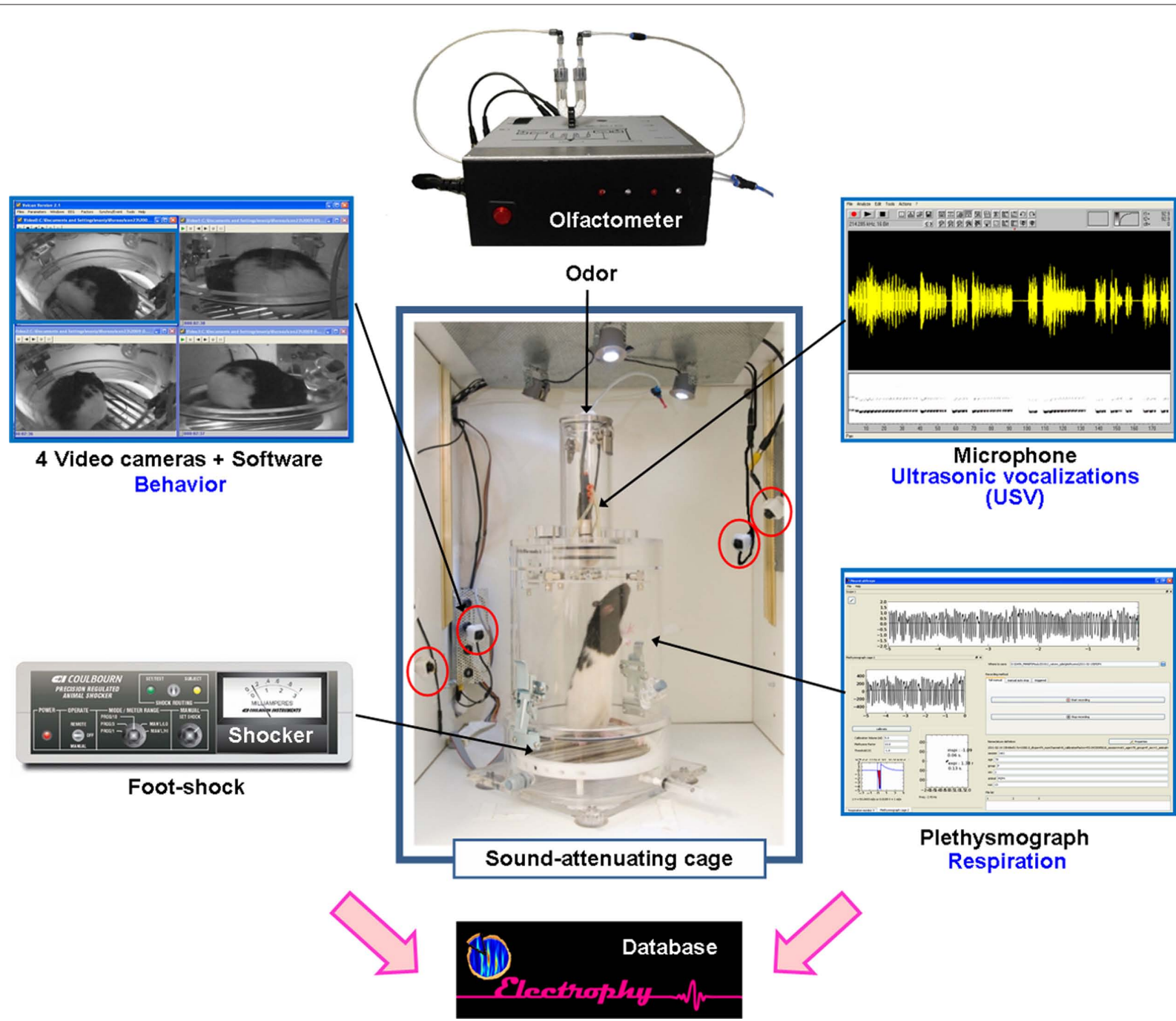

FIGURE A1 | Illustration of the different components of the RUB cage. A customized plethysmograph (central picture) was used to measure respiratory activity in behaving rats. The ceiling of the plethysmograph was equipped with a tower allowing the introduction of odorants delivered from an olfactometer. An ultrasound microphone was inserted in the tower to monitor ultrasonic vocalizations (USV) emitted by the rats. Animals' behavior was monitored using four video cameras placed at each corner of the sound-attenuating cage. The bottom of the plethysmograph was equipped with a floor connected to a shocker to deliver foot-shocks. The collected data were entered in a database (OpenElectrophy) for offline synchronization and analysis. 